\title{
Research of Information Recovery Methods Corresponding to Up-shift Part in NB-PLC
}

\author{
Chao Li ${ }^{1, ~ *, ~ Y o n g j i a n ~ J i n g ~}{ }^{1,2}$ \\ ${ }^{1}$ Inner Mongolia Xinyuan Information Technology Co., LTD, Huhhot, China \\ ${ }^{2}$ Center of Information and Network Technology, Inner Mongolia Agricultural University, Huhhot, China
}

Email address:

Digita19898@sina.com (Chao Li),wshbupt@163.com (Yongjian Jing)

*Corresponding author

\section{To cite this article:}

Chao Li, Yongjian Jing. Research of Information Recovery Methods Corresponding to Up-shift Part in NB-PLC. American Journal of Networks and Communications. Vol. 5, No. 2, 2016, pp. 46-50. doi: 10.11648/j.ajnc.20160502.15

Received: March 7, 2016; Accepted: April 12, 2016; Published: June 2, 2016

\begin{abstract}
Power line communication (PLC) is applied in various areas, such as Internet access, smart home, automatic meter reading system, security monitoring system, etc. The introduction of Orthogonal Frequency Division Multiplexing (OFDM) improves the reliability and efficiency of PLC substantially, and the standards of wide-band PLC and narrow-band PLC are becoming more complete. This paper is based on the receiver designing work according to the transmitter flow described in ITU-T G.9955. Regarding to the up-shift part of transmitter, two different methods to recover the useful information are provided including Hilbert transform and sub-carrier calculation. Both the theoretic analysis and simulation result shows that the two methods can recover the transmitted data exactly, while the second method can decrease the complexity of receiver and improve the efficiency of PLC system greatly.
\end{abstract}

Keywords: Narrow-Band Power Line Communication, G.9955, Up-shift, Hilbert Transform, Sub-carrier Calculation

\section{Introduction}

Power Line Communication (PLC) technology is widely distributed, coaxial cable and telephone lines are used as a transmission medium for data transmission and voice communications, also widely used in the last kilometer broadband access in remote areas. According to the different band, PLC is usually divided into broadband PLC (1.8 $250 \mathrm{MHz})$ and narrowband PLC $(3 \sim 500 \mathrm{KHz})$. Broadband power line communications used for broadband data and multimedia signal transmission, and narrowband power line communication provides a relatively low transmission rate, used for transmission of control information in smart grid and industrial control.

The first generation of narrowband power line communication using single-carrier FSK or PSK modulation, the transmission rate is low, and with low anti-interference ability. With the introduction of OFDM (Orthogonal Frequency Division Multiplexing) technology, the second-generation narrowband PLC is formed. The main standards include G3-PLC standard and PRIME standards. In early 2010, to make the product worldwide, P1901.2 and G.9955 (G.hnem physical layer specification) are promoted by IEEE and ITU-T separately, this paper is based on research of G.9955.

According to standard G.9955 [1], NB-PLC frequency band is divided into two categories, one is CENELEC (European Committee for Electro technical Standardization) band $(3 \mathrm{KHz}-148.5 \mathrm{KHz})$, the other is FCC band $(9 \mathrm{KHz}-490 \mathrm{KHz})$.

\section{The PLC Transmit System}

Narrow band PLC system use OFDM modulation to transmit information. It's physical frame including Preamble, $\mathrm{PFH}, \mathrm{CES}$ and payload sections, as shown in figure 1. Preamble and CES does not carry any information, the function is synchronization and channel estimation, and transmit data is in payload section.

\begin{tabular}{|l|l|l|l|l}
\hline Preamble & PFH & CES & PFH & Payload \\
\hline
\end{tabular}

Figure 1. The frame structure. 
With Different number of symbols in PFH, the modulation and coding scheme is different. Payload length may vary in different frame, the number and bandwidth of subcarriers in each OFDM symbol is shown in table 1.

Table 1. OFDM parameter

\begin{tabular}{lll}
\hline Variable & parameter & value \\
\hline $\mathrm{N}$ & Number of subcarriers & $2 \mathrm{k}, \mathrm{k}=7,8$ \\
FSC & Bandwidth of subcarriers $[\mathrm{KHz}]$ & $15.625 / \mathrm{n}, \mathrm{n}=5,10$ \\
FUS & Shift frequency $[\mathrm{KHz}]$ & $(\mathrm{N} / 2)^{*} \mathrm{FUS}$ \\
\hline
\end{tabular}

In different band of CENELEC and FCC, the number of subcarriers in each OFDM symbol is different.

Table 2. OFDM subcarriers in different band.

\begin{tabular}{|c|c|c|c|c|}
\hline Band & Start frequency & End frequency & Number of subcarriers & Empty subcarriers \\
\hline CENELEC-A & $35.9375 \mathrm{KHz}$ & $90.625 \mathrm{KHz}$ & $23 \sim 58$ & $0-22,59-127$ \\
\hline CENELEC-B & $98.4375 \mathrm{KHz}$ & $123.4375 \mathrm{KHz}$ & $63 \sim 79$ & $0-62,80-127$ \\
\hline CENELEC-CD & $125 \mathrm{KHz}$ & $143.75 \mathrm{KHz}$ & $80 \sim 92$ & $0-79,93-127$ \\
\hline FCC & $34.375 \mathrm{KHz}$ & $478.125 \mathrm{KHz}$ & $11 \sim 153$ & $0-10,154-255$ \\
\hline FCC-1 & $34.375 \mathrm{KHz}$ & $137.5 \mathrm{KHz}$ & $11 \sim 43$ & $0-10,45-255$ \\
\hline FCC-2 & $150 \mathrm{KHz}$ & $478.125 \mathrm{KHz}$ & $48 \sim 153$ & $0-47,154-255$ \\
\hline
\end{tabular}

The transmit process of PLC system is shown in figure 2.

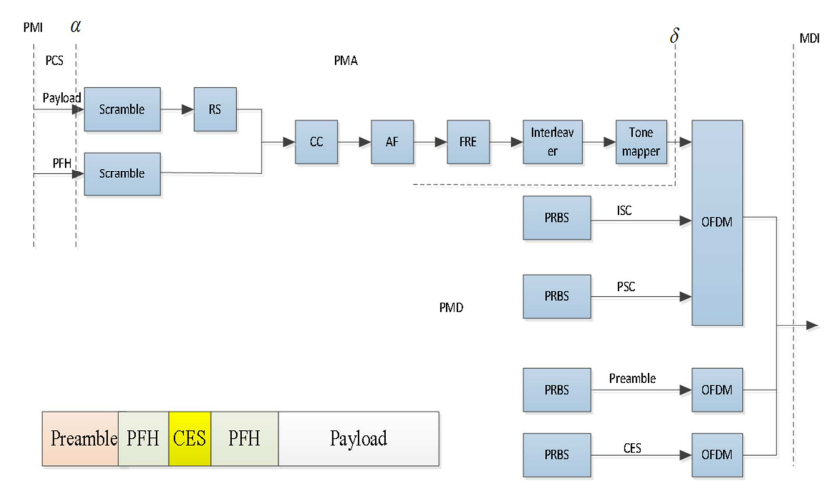

Figure 2. The transmit process.

As shown in figure 2, the payload data is the research focus. At the transmitting end, the data need to be transmitted first perform scrambling, using pseudo-random sequence generated by shift register (LFSR) [2-5]. then forward error correction coding (FEC), including RS coding and convolution coding (CC), then the packet aggregation (AF), block repetition coding, interleaving and channel ranks to improve data anti-interference performance.

In a typical modulation technique for link-level system, the real part of the OFDM symbol is transmitted directly, and receive end can use oversampling and Hilbert transform to recover data [6-7]. In G.9955 standard, the transmitter-side first oversampling OFDM symbol, then frequency shift, and then take the real part into the channel for transmission, as shown in figure 3 .

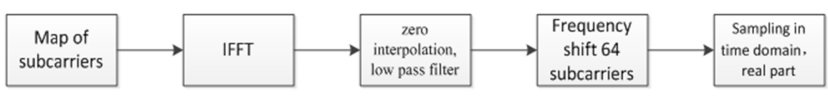

Figure 3. The transmit end.

In OFDM modulation first turn the frequency domain discrete points $\mathrm{N}$ (128 or 256 ) into time-domain signal by IFFT transform. For example, just take a point in OFDM symbol. Set the points in the frequency domain as

$$
\mathrm{X}(k, m)=a_{m}+j b_{m}(0 \leq m \leq N-1)
$$

Get $\mathrm{N}$ discrete time-domain signal sample points by IFFT.

$$
\begin{aligned}
& u_{k}(n)=\operatorname{IFFT}(X(k))=\frac{1}{N} \sum_{m=0}^{N-1}(a(k, m)+j b(k, m)) e^{j \frac{2 \pi m n}{N}} \\
& \frac{1}{N} \sum_{m=0}^{N-1}\left\{a(k, m) \cos \left(2 \pi \frac{m n}{N}\right)-b(k, m) \sin \left(2 \pi \frac{m n}{N}\right)\right\}+ \\
& j \frac{1}{N} \sum_{m=0}^{N-1}\left\{a(k, m) \sin \left(2 \pi \frac{m n}{N}\right)+b(k, m) \cos \left(2 \pi \frac{m n}{N}\right)\right\},(n=0,1, \ldots N-1)
\end{aligned}
$$

Perform $p\left(p=2^{\alpha}\right)$ oversampling to time domain sampling points, get discrete data.

$$
\begin{aligned}
& u_{k}(n)= \\
& \frac{1}{N} \sum_{m=0}^{N-1}\left\{a(k, m) \cos \left(2 \pi \frac{m n}{p N}\right)-b(k, m) \sin \left(2 \pi \frac{m n}{p N}\right)\right\}+ \\
& j \frac{1}{N} \sum_{m=0}^{N-1}\left\{a(k, m) \sin \left(2 \pi \frac{m n}{p N}\right)+b(k, m) \cos \left(2 \pi \frac{m n}{p N}\right)\right\},(n=0,1,2 \ldots p N-1)
\end{aligned}
$$

Performs frequency shift operation $F_{U S}=m \times F_{S C}$, where $\mathrm{m}$ is an integer no less than $\mathrm{N} / 2, \mathrm{G} .9955$ specified $\mathrm{m}=\mathrm{N} / 2$, after the frequency shift, time domain sampling point becomes:

$$
s_{n}=u_{k}(n) e^{j \frac{2 \pi m n}{N p}}=\operatorname{Re}\left(s_{n}\right)+j \operatorname{Im}\left(s_{n}\right),(0 \leq n \leq 2 N-1)
$$

Transmit signal is the real part of $s_{n}$ :

$$
s_{\text {out }}=\operatorname{Re}\left(s_{n}\right)=\operatorname{Re}\left(u_{k}(n)\right) \cos \left(\frac{2 \pi m n}{N p}\right)-\operatorname{Im}\left(u_{k}(n)\right) \sin \left(\frac{2 \pi m n}{N p}\right)
$$

\section{Two Recover Solutions}

Two ways are proposed to recover the original data respectively, one is the Hilbert transform detection algorithms; the other is subcarriers calculation solution according to the 
frequency domain.

\subsection{Hilbert Transform Solution}

At the receiving end, first test the received data, then recover the frequency-domain signal $X(k, m)$.

Take $p\left(p=2^{\alpha}\right)$ and $\mathrm{m}=\mathrm{N} / 2$ into receive signal

$r_{n}=s_{\text {out }}=\operatorname{Re}\left(u_{k}(n)\right) \cos \left(\frac{n \pi}{2^{\alpha}}\right)-\operatorname{Im}\left(u_{k}(n)\right) \sin \left(\frac{n \pi}{2^{\alpha}}\right),(0 \leq n \leq p N-1)$

When $n=2^{\alpha} i(i=0,1,2 . . N-1)$

$$
r_{2 i}=\operatorname{Re}\left(u_{k}\left(2^{\alpha} i\right)\right) \cos (i \pi)=(-1)^{i} \operatorname{Re}\left(u_{k}\left(2^{\alpha} i\right)\right)
$$

When $n=\left(i+\frac{1}{2}\right) \cdot 2^{\alpha}(i=0,1,2 . . N-1)$

$$
\begin{aligned}
& r_{2 i+1}=-\operatorname{Im}\left(u_{k}\left(2^{\alpha}\left(i+\frac{1}{2}\right)\right)\right) \sin \left(\frac{(2 i+1) \pi}{2}\right) \\
& =(-1)^{i+1} \operatorname{Im}\left(u_{k}\left(2^{\alpha}\left(i+\frac{1}{2}\right)\right)\right)
\end{aligned}
$$

The Hilbert transform [3]is:

$$
\tilde{f}(t)=H[f(t)]=\frac{1}{\pi} \int_{-\infty}^{\infty} \frac{f(\tau)}{t-\tau}=f(t) \otimes \frac{1}{\pi t}
$$

According to the define of resolve signal,

$$
f_{a}(t)=f(t)+\tilde{j}(t)
$$

By equation (3), $u_{k}(n)$ is resolve signal, the imaginary part is the Hilbert transform of the real part, get all real part of sampling points of (N) (as in formula (7)), and imaginary part (such as the formula (8)) of $\mathrm{N}$ over-sampling points. With inverse Hilbert transform of imaginary part, real part of $2 \mathrm{~N}$-point can obtained.

$$
r_{2 i+1}{ }^{\prime}=H^{-1}\left(r_{2 i+1} \cdot(-1)^{i+1}\right)
$$

Connect these real part point, get $r_{i}=\left(r_{0}, r_{1}{ }^{\prime}, r_{2}, r_{3}{ }^{\prime} \ldots\right)(i=0,1,2 \ldots .2 N-1)$, perform $2 \mathrm{~N}$-point FFT transform, derived the original frequency domain signal.

$$
\begin{aligned}
& y_{l}=\sum_{n=0}^{2 N-1} r_{n} e^{-j \frac{2 \pi n l}{2 N}}=\sum_{n=0}^{2 N-1}\left\{\frac{1}{N} \sum_{m=0}^{N-1}\left[a(k, m) \cos \left(2 \pi \frac{m n}{2 N}\right)-b(k, m) \sin \left(2 \pi \frac{m n}{2 N}\right)\right]\right\} e^{-j \frac{2 \pi n l}{2 N}} \\
& ==\left\{\begin{array}{cc}
2 a(k, 0) & 1=0 \\
a(k, l)+j b(k, l) & 1 \leq l \leq N-1 \\
\text { other } & N \leq l \leq 2 N-1
\end{array}\right.
\end{aligned}
$$

Thus, in addition to the first data restoring the real part, the other N-1 point complex data can be recovered. As no data was put in the first sub-carrier, there is no impact on the demodulation of received data.

\subsection{Subcarriers Calculation Solution}

In this section, after the analysis of frequency domain change, can get information from received signal by FFT transform, at different locations of subcarriers.
After modulation, transmit signal was mapped to the corresponding sub-carriers, for example, CENELEC A band use No.24-59 subcarriers, CENELEC B use No.64-80 subcarriers, CENELEC CD use No.81-93 subcarriers. Then perform oversampling and interpolation. When the interpolation factor is $\mathrm{p}$, insert ( $\mathrm{p}-1)$ zeros between every two time domain sampling point, corresponding to changes in the frequency domain of the original signal, which is called mirroring, the process is shown in figure 4 .

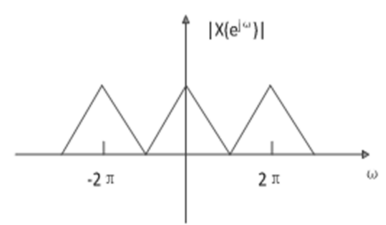

(a) original spectrum

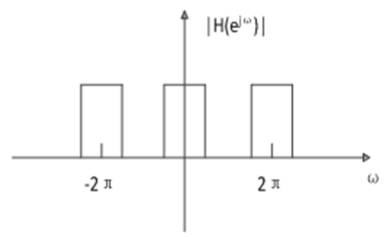

(a) spectrum of low pass filter

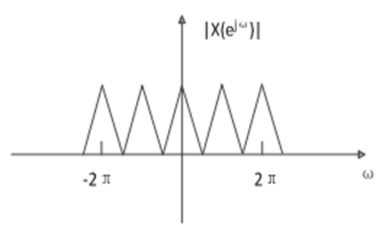

(b) mirror of zero interpolation $(\mathrm{p}=2)$

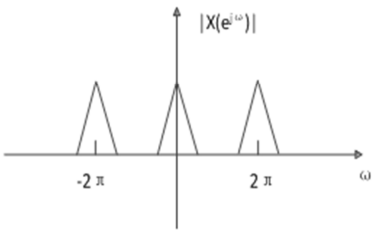

(b) spectrum of sampling signal
Figure 4. The mirroring process.

After over-sampling, move $m \geq N / 2$ subcarriers, then take the real part, replicate the spectrum on the complex frequency domain again, and conjugate.

Take CENELEC A band as an example; put data in 24-59 subcarriers, as shown in figure 5 .

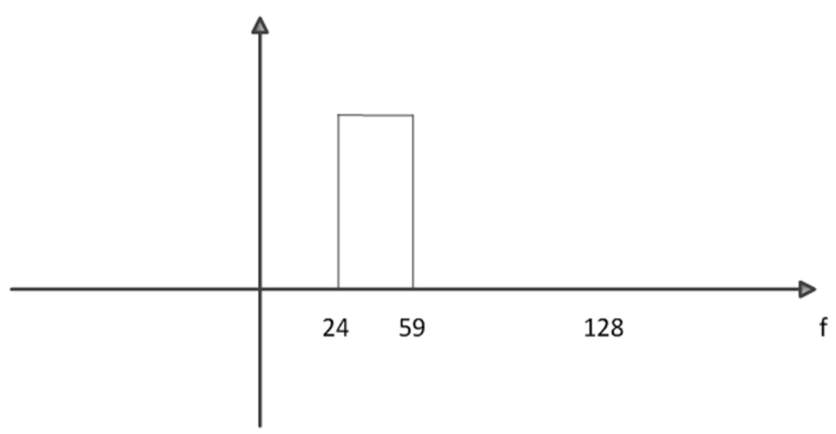

Figure 5. Data of CENELEC A.

Then zero interpolation, low pass filter.

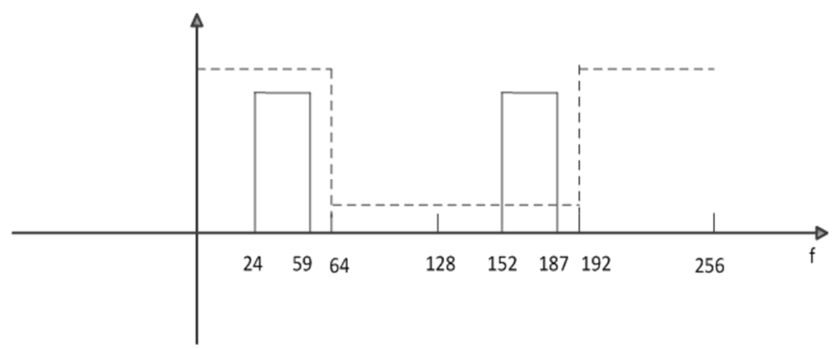

Figure 6. Spectrum of oversampling. 
As shown in figure 6, the dotted line is shape of filter, data in 24-59 sub-carriers can pass, and the data in subcarrier 155-187 will be filtered out.

Then is the frequency shift, move 64 subcarriers:

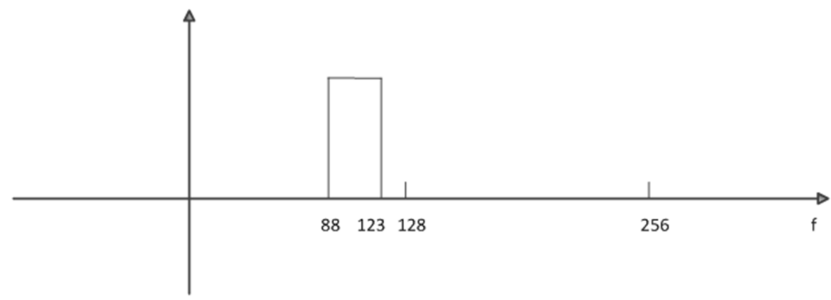

Figure 7. Spectrum of shift.

And the real part is

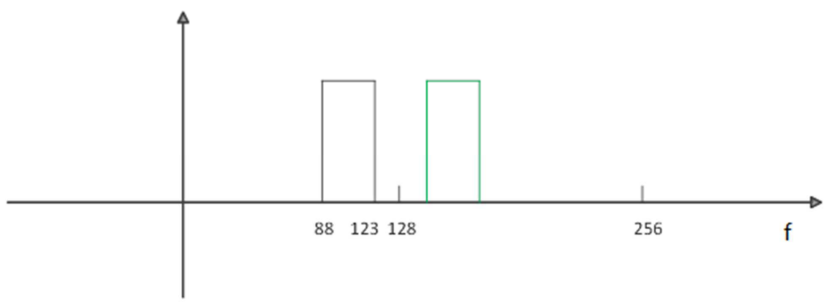

Figure 8. Spectrum of real part.

The green part is the conjugate of the original data, that is the negative frequency and positive frequency spectrum is symmetric.

In summary, in the receiving end, get $88-123$ point out, which is the data required.

The change process of CENELEC $\mathrm{B}$ is as follows:

First, data is placed in 64-80 subcarriers, shown in figure9.

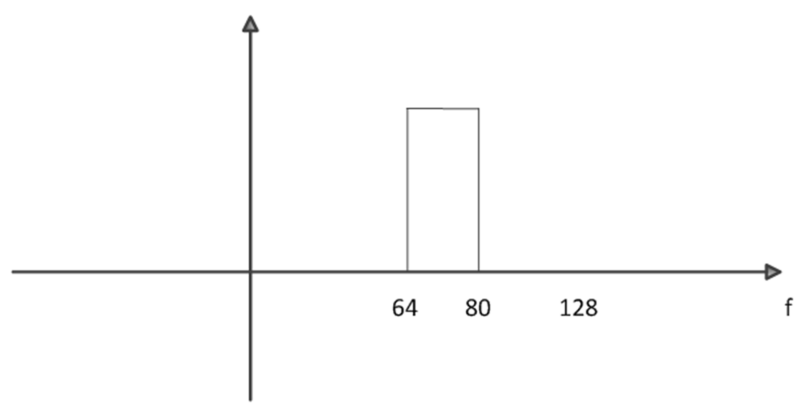

Figure 9. Data placed in 64-80 subcarriers.

Then, zero interpolation and low-pass filter, through the low-pass filter, the data in 192-208 is reserved; data in 64-80 is filtered, as shown in figure 10 .

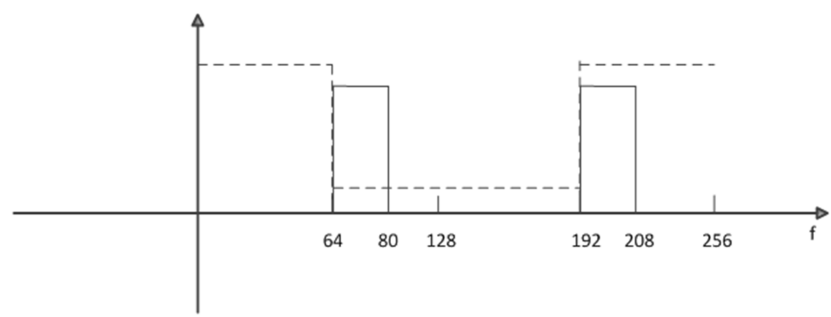

Figure 10. Low pass filter.
Then moved 64 sub-carrier frequency, shown in figure 11.

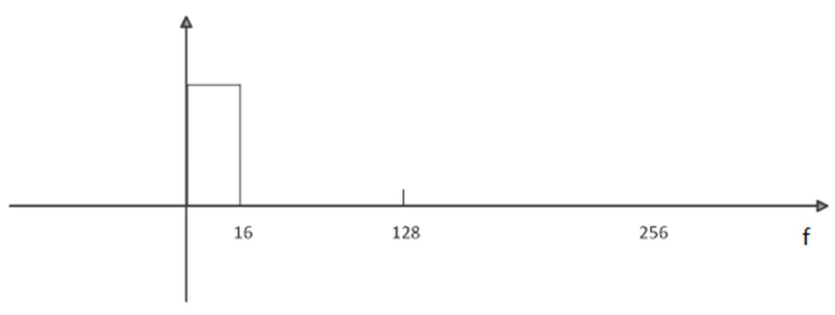

Figure 11. 64 frequency move.

Take real part, get figure 12 .

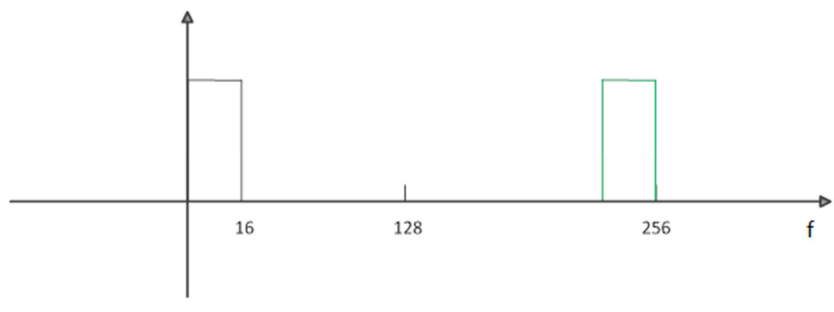

Figure 12. Real part.

CENELEC CD similar to CENELEC B, after a low-pass filter, the data in 128-256 is retained, while others is filtered, and then frequency shift, the data bits back to the first 128 sample points. Transmitted data is located on the positive frequency spectrum regardless of band.

Therefore, the receiving end can calculate subcarriers occupied by the data, take the data from corresponding sub-carriers to operate, without Hilbert transform.

\section{Simulation Results and Conclusion}

From the above analysis, the two solutions didn't process the noise and interference in the subcarriers, they should have same performance. Simulation platform was setup to verify the solutions, the receiver process is shown in figure13.

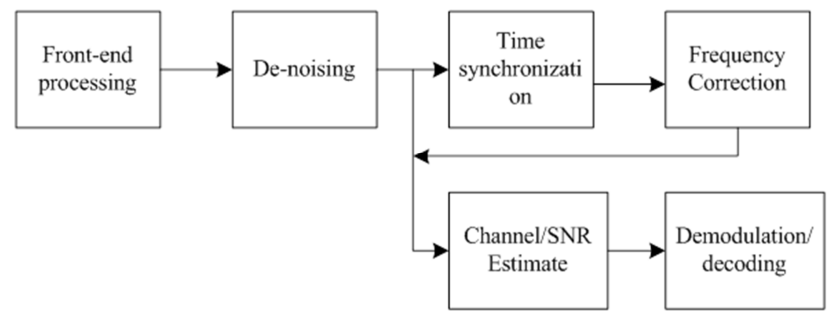

Figure 13. The receiver process.

As shown in figure 13, the receiving end is the reverse process of the transmitting end, the receiving end perform demodulation and decoding. The de-noising module includes interference filter in narrowband, and against burst; time synchronization includes coarse synchronization and fine synchronization; decoding includes convolution decoding and RS decoding.

There are five kind of noise in PLC channels, including colored background noise, narrowband interference, periodic 
impulse noise, asynchronous random impulse noise and periodic random impulse noise [8-10]. Gaussian white noise is set in the channel, use the CENELEC A band to transmit data, each OFDM symbol has 128 sub-carriers, No.23-58 sub-carriers are used to transmit data. The performance comparison of the two solutions is shown in figure 14 .

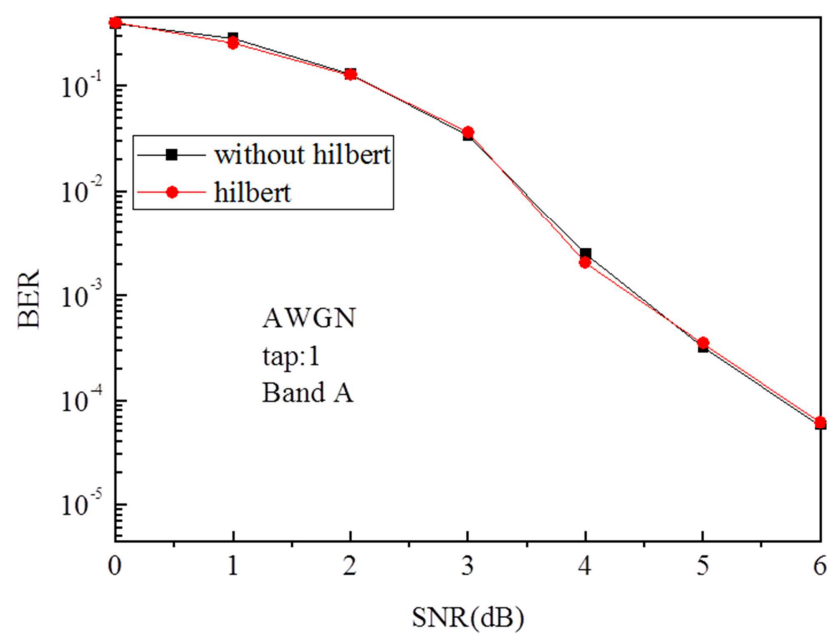

Figure 14. Comparison of Hilbert transform and subcarrier computing.

In the above figure, the red line is the Hilbert transform performance curve, the black line is subcarrier computing performance curve. The two solutions, whether theoretical analysis or link-level simulation, can recovery original data, and no difference in performance. But the sub-carriers calculation solution can filter data from frequency domain directly, and quickly, which can greatly improve the system efficiency.

\section{References}

[1] ITU-T G.9955,Narrowband OFDM Power Line Communication Transceivers-Physical Layer Specification, 2011. 12.

[2] Xiaoyu Wen, Oversampling and Hilbert transform in DAMB system. Journal of Nan yang Normal university. 2008.3.

[3] Jiongpan Zhou, The principle of communication, pp 27-31.

[4] Xiuqing yuan, Study of channel estimation in PLC based on OFDM. Harbin University of Science and Technology, 2008.

[5] Stefania Sesia, Issam Toufik, Matthew Baker. LTE-The UMTS Long Term Evolution From Theory to Practice. Pp 91.

[6] J. Maxwell, A Book on Electricity, 9rd ed., Oxford: Clarendon, 1892, pp. 88.

[7] J. Young, A Technical Write, 5rd ed., vol. 9. Oxford: Clarendon, 2002, pp. 73.

[8] Feng Ye, Study of PLC simulation, Xinan Jiaotong University, 2008.

[9] Jianhua Zhao, Zhi Li, Simulation of OFDM in PLC system, Computer and Digital Engineer, 2011, Vol. 39, No. 9.

[10] V. Degardin, M. Lienard and P. Degauque et al, "Impulsive noise on indoor power lines: characterization and mitigation of its effect on PLC systems", Electromagnetic Compatibility, IEEE, May 2003, vol. 1, pp. 166-169. 